\title{
Repères et enjeux
}

Jean Duverger

\section{(2) OpenEdition}

Journals

Édition électronique

URL : http://journals.openedition.org/ries/3942

DOI : $10.4000 /$ ries.3942

ISSN : 2261-4265

\section{Éditeur}

Centre international d'études pédagogiques

\section{Édition imprimée}

Date de publication : 7 septembre 1995

Pagination : 29-44

ISSN : 1254-4590

\section{Référence électronique}

Jean Duverger, «Repères et enjeux », Revue internationale d'éducation de Sèvres [En ligne], 07 | 1995, mis en ligne le 02 octobre 2014, consulté le 01 mai 2019. URL : http://journals.openedition.org/ ries/3942 ; DOI : 10.4000/ries.3942

Ce document a été généré automatiquement le 1 mai 2019.

(c) Tous droits réservés 


\title{
Repères et enjeux
}

\author{
Jean Duverger
}

1 C'est évidemment une banalité que d'affirmer que le monde de l'éducation ne peut être tenu à l'écart des évolutions politiques, économiques et culturelles de la fin du $\mathrm{XX}^{\mathrm{e}}$ siècle. Qu'il s'agisse en effet de la construction de l'Europe ou de la mise en place d'autres instances économiques plus vastes telles que l'Accord général sur les tarifs douaniers et le commerce (General Agreement on Tariffs and Trade, GATT), chacun constate forcément que la mondialisation des échanges, l'internationalisation de l'économie de marché, la multiplication des moyens de communication, ont pour effet de produire des besoins éducatifs nouveaux; les futurs adultes de cette fin de siècle et du siècle à venir ont en effet conscience de l'impérieuse nécessité de mieux comprendre "l'étranger ", toujours plus proche. Communiquer avec cet étranger omniprésent, pour lui vendre ou acheter des marchandises, mais aussi et surtout pour mieux le connaitre et vivre avec lui, c'est bien sûr lui parler, lui écrire, et l'école est alors interpelée dans sa mission d'apprentissage des langues, au-delà de l'indispensable maîtrise de la langue maternelle. Alors l'enseignement bilingue est en vogue, c'est vrai, même si le concept est flou; même si, comme trop souvent, on demande à l'école bien plus qu'elle ne peut donner. Mais comment ne pas voir qu'il ne s'agit pas seulement d'un effet de mode, mais bien plutôt d'une tendance lourde, liée aux évolutions socio-économiques des sociétés, à l'échelle mondiale?

2 Nous tenterons, dans ce court article, de cerner d'abord le champ de l'enseignement bilingue, en esquissant quelques repérages. Puis nous illustrerons le fait que ce type d'enseignement est finalement assez répandu de par le monde, avant de décrire brièvement la situation en France, une situation difficile mais en pleine évolution. Nous tenterons de montrer ensuite que cet enseignement bilingue mériterait assurément un grand développement, tant les bénéfices potentiels peuvent en être importants et intéressants, mais à certaines conditions, conditions que nous nous efforcerons d'identifier et de décrire en conclusion. 


\section{Un essai de classification}

3 Au préalable, il convient d'être très clair au plan lexical : un enseignement bilingue est bien un système où l'enseignement est dispensé en deux langues.

4 En d'autres termes, il ne suffit pas d'inclure dans un cursus scolaire l'apprentissage même approfondi - d'une deuxième langue pour que ce cursus puisse être qualifié de bilingue.

5 Il y a explicitement enseignement bilingue lorsque les deux langues en présence sont " véhiculaires ", c'est-à-dire lorsqu'elles servent toutes les deux à apprendre des savoirs autres que linguistiques. Parmi ces deux langues l'une, première langue (L1), peut-être la langue maternelle de l'élève, mais pas forcément; et de toutes façons, une deuxième langue (L2) coexiste avec la première, en tant que langue d'apprentissage scolaire.

6 Mais cette nécessaire définition de l'enseignement bilingue recouvre bien sûr des réalités très diverses. De nombreux auteurs ont établi des typologies qui dépendent bien entendu des critères qu'ils se donnent pour les construire.

7 À titre d'exemple, citons Mackey ${ }^{1}$ qui dénombre quatre-vingt-dix types d'éducation/ enseignement bilingue en se basant sur l'emploi des deux langues dans leur distribution selon quatre dimensions : la famille, l'école, l'environnement et la nation.

8 Citons encore Hamers et Blanc ${ }^{2}$ qui préfèrent utiliser comme critère de classification des facteurs socio-historiques, socio-structuraux, culturels, idéologiques, aboutissant ainsi à une douzaine de modèles.

9 On peut aussi tout naturellement classer les différents systèmes scolaires en fonction des degrés de monolinguisme ou de bilinguisme qu'ils visent. Les objectifs linguistiques des puissances politiques varient en effet considérablement d'un pays à l'autre, et ils varient aussi à l'intérieur d'un même pays en fonction des régions et de l'histoire. Car on sait depuis bien longtemps que les langues sont des instruments de pouvoir, d'hégémonie et de domination, et que la fin de la " guerre des langues ${ }^{3}$ " n'est pas pour demain.

10 Pour prendre l'exemple de la France, chacun reconnaît que tous les efforts des pouvoirs politiques, de façon ininterrompue depuis Villers-Cotterêts (en passant par la Révolution française, Napoléon, et les lois Ferry de la République) ont tenté de construire une école monolingue, s'efforçant par conséquent d'éradiquer les autres langues du territoire. On retrouve les mêmes phénomènes en Espagne, en Italie ou en Grande-Bretagne, pour rester dans le champ de l'Union européenne. Et si maintenant, depuis une ou deux décennies, on réhabilite les langues dites régionales ${ }^{4}$, cela conduit à des types d'organisation scolaire qui essaient de s'adapter au mieux aux objectifs linguistiques visés. D'où les trois systèmes scolaires basques espagnols $(\mathrm{A}, \mathrm{B}, \mathrm{D})$, les très nombreuses catégories d'immersion canadienne ${ }^{5}$, les diverses solutions qui commencent à se développer en Suisse, pays officiellement plurilingue, mais peuplé, jusqu'à présent, d'une majorité de monolingues.

11 D’une façon générale, répétons-le, les typologies dépendent donc bien des critères retenus. Nous choisirons pour notre part de nous situer ici dans une perspective pédagogique, dans le cas où un pouvoir politique a décidé de mettre en place un dispositif scolaire de nature à développer un bilinguisme fonctionnel et efficace. Quels critères alors choisir, quels paramètres doivent être privilégiés? 
12 Nous sommes ici dans l'ordre du "comment faire", ce qui permettra d'esquisser une autre typologie et de donner d'autres repères. Les critères retenus sont au nombre de quatre, décrivant des dispositifs scolaires fort contrastés. Ils répondent à des questions simples du type : quand introduire la seconde langue? De quelle manière? Avec quelle répartition entre la première et la seconde langue? Quels temps respectifs?

\section{Le moment de l'introduction de la seconde langue dans la scolarité de l'élève}

Ou bien l'enseignement est bilingue dès le début du cursus scolaire (Val d'Aoste, Luxembourg, certaines classes bilingues en Alsace ou au Pays Basque français) Ce sont là des modèles de bilinguisme précoce (le terme précoce ne convient vraiment que si la langue 2 est démarrée dès la maternelle).

Ou bien l'enseignement est bilingue seulement à partir de sept, huit, neuf ou dix ans (fin du cycle élémentaire). Ce sont des cas de bilinguisme scolaire intermédiaire, comme dans certaines écoles québécoises ou d'Europe du Nord, mais aussi les écoles européennes et les sections internationales françaises.

15 Ou bien l'enseignement n'est bilingue qu'à partir du second degré, après un apprentissage de la langue 2 plus ou moins intense. C'est là un bilinguisme scolaire tardif, comme en Tunisie par exemple, ou certains lycées allemands bilingues; c'est le cas aussi des lycées bilingues polonais, hongrois, bulgares ou vietnamiens. C'est enfin le cas des sections européennes, en France.

\section{La manière dont est introduite la deuxième langue dans le cursus scolaire}

Ou bien cette introduction est " progressive », avec des horaires réguliers d'enseignement de seconde langue, préparant l'enseignement bilingue proprement dit. On apprend la langue 2 pendant deux, trois ou quatre ans (quatre ans par exemple en Tunisie, à raison de dix à douze heures par semaine) avant de s'en servir comme langue véhiculaire: le présupposé est alors qu'il faut apprendre une langue avant de l'utiliser, ce qui, on le sait, est très contestable.

Ou bien cette introduction est «brutale », et la langue 2 est, très vite, après une année, voire immédiatement, une langue véhiculaire.

C'est le cas des différents modèles d'immersion, imaginés au Québec au début des années soixante, pour un public anglophone vivant dans un environnement majoritairement francophone (Montréal) et dont Lambert et son équipe ont popularisé les résultats ${ }^{6}$.

Un cas assez proche est celui des écoles françaises à l'étranger où, dès l'école maternelle, des petits nationaux sont immergés scolairement dans une langue qui n'est pas la leur, une L2 qui devient immédiatement langue d'apprentissage 1. Notons toutefois que des différences existent entre l'immersion canadienne et ces cas d'écoles françaises hors de France, où les petits nationaux vivent, si l'on n'y prend garde, plutôt une sorte de submersion qu'une immersion maîtrisée et régulée.

Une troisième manière d'introduire une seconde langue est utilisée dans un certain nombre de pays dits de l'Est, comme la Russie, la Pologne, la Bulgarie : au début de 
l'enseignement secondaire (onze, douze, treize ans) et pendant une année entière, il y a immersion brutale - mais par conséquent tardive - en langue 2 sans que pour autant on démarre des apprentissages disciplinaires dans cette L2. Et après cette année d'intense apprentissage de L2 (dite année 0) on démarre l'enseignement réellement bilingue.

\section{La répartition des matières entre langue 1 et langue 2}

21 Ce problème de la place et du lieu des deux langues dans les programmes est évidemment lourd de significations, et l'on peut, à partir de ce paramètre, distinguer plusieurs cas de figure.

Ou bien les matières sont indifféremment enseignées en première ou seconde langue, avec des variations selon les années du cursus scolaire, selon les sujets, mais sans privilégier une langue pour telle ou telle discipline (Val d'Aoste). Ce bilinguisme scolaire " équilibré » est souhaité dans certaines régions où deux langues coexistent en parité, mais cet équilibre est toujours fragile, en raison de la fragilité même des statuts de langue (voir la Catalogne).

23 Ou bien des matières sont plus spécifiquement enseignées en langue 1 , d'autres en langue 2 (cas les plus fréquents) et en fonction de critères dont il serait intéressant de faire une analyse fine. Il en est ainsi en Tunisie (seules les matières scientifiques sont enseignées en français dans le secondaire) ou dans les sections bilingues françaisallemand de Rhénanie du Nord-Westphalie (là, ce sont les matières littéraires et surtout l'histoire-géographie qui sont enseignées en français).

\section{La répartition des temps horaires impartis aux enseignements en langue 1 et langue 2}

Cet aspect quantitatif n'est pas négligeable, et peut également renvoyer à des types divers d'enseignement bilingue.

En Alsace, où l'enseignement bilingue progresse significativement, trois formules existent actuellement dénommées 3, 6, 13, les chiffres désignant le nombre d'heures en langue 2.

Les programmes américains d'enseignement bilingue (très nombreux et très à la mode) se distinguent entre eux par les pourcentages respectifs de langue 1 et langue 2 (on parle de programme $80 / 20$ ou $70 / 30$ et des cabinets d'experts sont censés proposer la formule la mieux adaptée à la « demande-commande »).

Le Val d'Aoste, pour sa part, a opté pour un programme strictement équilibré, de même que les classes élémentaires bilingues du Pays basque français (français-basque). Le Pays basque espagnol a également quelques classes bilingues 5 0/50. Il en existait aussi beaucoup en Catalogne espagnole, mais la catalanisation s'accélère dans les classes primaires, si bien que la parité scolaire linguistique est en voie de disparition.

Comme on le voit, par conséquent, et seulement en fonction de ces quatre paramètres, de nombreux modèles d'enseignement bilingue peuvent être distingués au plan des stratégies pédagogiques. Mais on pourrait à loisir multiplier des cas de figure, en mettant en relation ces modèles avec d'autres paramètres, importants également, comme peuvent l'être le choix des langues, la distance linguistique entre ces langues (proches ou lointaines), l'articulation pédagogique entre langue 1 et langue 2 , le choix et la 
spécialisation - ou non - des maîtres, les mesures d'accompagnement et, surtout, les statuts de langue 1 et langue 2 dans la société et/ou dans le vécu de l'élève/apprenant.

Les systèmes d'enseignement bilingue sont donc complexes et labiles, très dépendants d'abord de l'histoire, de la sociologie, de la situation linguistique des pays qui les mettent en œuvre. Le croisement de ces données avec des considérations d'ordre économique et pédagogique aboutissent à des dispositifs scolaires infiniment variés que nous avons essayé d'ordonner. Les propos qui suivent illustreront quelque peu les disparités, sans prétendre à l'exhaustivité.

\section{Fluctuations et contrastes}

Les trois quarts, ou plus, des habitants de la planète sont bilingues à des degrés divers, qu'il s'agisse de bilinguisme familial (enfants de "couples mixtes", selon l'expression consacrée), de bilinguisme "migratoire» (migrations économiques, exil) ou de bilinguisme « fortuit » (longs voyages, rencontres amoureuses...) ; les bilingues, mais aussi trilingues, etc., sont infiniment plus nombreux que les monolingues; c'est tout à fait incontestable.

31 Mais il n'y a pas forcément corrélation, dans un pays, entre ce bilinguisme de fait et les objectifs linguistiques du système éducatif de ce pays.

32 En d'autres termes, il arrive souvent que des gens deviennent bilingues malgré leur système éducatif, en marge de celui-ci, voire même en opposition avec lui. L'inverse existe aussi d'ailleurs, et n'est pas sans poser problème, à savoir qu'un système éducatif peut avoir pour objectif explicite de former des individus bilingues sans y parvenir vraiment.

33 C'est que la langue s'apprend - ou ne s'apprend pas - aussi (surtout ?) à l'extérieur de l'école, c'est qu'elle est l'expression de pouvoirs politiques et de dominances économiques, comme nous l'avons souligné ci-dessus. C'est enfin qu'elle a pour chaque individu un sens, un statut particulier, liés à son identité et son histoire personnelle. Et, en définitive, l'apprentissage d'une langue est très corrélé à la représentation que l'apprenant se fait de cette langue.

Indépendance, par conséquent, entre la situation linguistique d'un pays et les objectifs linguistiques de son système éducatif. Fluctuations et contrastes, variabilité en fonction de paramètres géopolitiques et économiques, telles semblent être les caractéristiques essentielles de l'enseignement bilingue dans le monde. Sans doute le bilinguisme en général, le bilinguisme scolaire en particulier, sont-ils de plus en plus "recherchés » au niveau mondial, et les cas de monolinguisme triomphant, de nature idéologique (Hitler ne disait-il pas que la langue allemande était une langue supérieure ?) sont peut-être en recul. Mais les rechutes sont toujours possibles, racisme et nationalisme aidant.

Nous évoquerons rapidement quelques exemples de par le monde, proches de la France d'abord, puis appartenant à des pays plus éloignés, illustrant ainsi l'extrême variété des bilinguismes scolaires.

\section{Le Luxembourg, pays de trilinguisme}

Le système éducatif luxembourgeois est organisé de telle façon que tous les élèves soient au moins trilingues : luxembourgeois, allemand, français. Les langues se succèdent dans le 
cursus scolaire (d'abord luxembourgeois, puis allemand, enfin français) et elles sont spécialisées (la langue luxembourgeoise étant essentiellement orale). Le modèle luxembourgeois prouve que le trilinguisme n'est pas inaccessible, qu'il ne rend pas plus idiot que le bilinguisme ou le monolinguisme ${ }^{7}$.

\section{Le Val d'Aoste, un bilinguisme institutionnel}

Depuis 1988, dans tous les établissements publics scolaires valdôtains, le français et l'italien sont déclarés «à égalité dans le temps d'enseignement ». Depuis la première année de maternelle, les élèves vivent leur scolarité en deux langues, et cette expérimentation en vraie grandeur a fait l'objet de descriptions passionnantes, comme celle de Rita Decîme ${ }^{8}$. Des évaluations commencent à être disponibles, avec des résultats qualifiés de très satisfaisants9.

\section{La Catalogne et le Pays basque espagnol, des bilinguismes volontaristes}

Avec la Constitution de 1978, des lois de normalisation linguistique ont été promulguées dans ces deux régions de l'Espagne, et des systèmes éducatifs originaux ont été mis en place, développant systématiquement un bilinguisme scolaire.

Même si de grandes différences existent dans les résultats de ces politiques (le catalan est beaucoup plus proche du castillan/espagnol que ne l'est le basque), on ne peut nier que ces politiques commencent à porter leurs fruits : la population bilingue est de plus en plus nombreuse. Les formules pédagogiques sont diverses, les difficultés sont fréquentes, des conflits se développent aussi, notamment en Catalogne, depuis précisément que les responsables linguistiques imposent une catalanisation totale à l'école élémentaire, abandonnant d'une certaine façon le bilinguisme scolaire $n$. Mais il reste que ces régions ont retrouvé leur identité linguistique, et les nombreuses stratégies utilisées ont utilement nourri la recherche pédagogique en matière d'apprentissage de langues. On pourrait aussi citer la Galice, le Pays de Galles, dans ce premier groupe d'exemples de bilinguisme scolaire de pays voisins. Partout la même volonté, dans ces cas de figure, de retrouver des langues régionales que les pouvoirs centraux tentaient d'éliminer voici seulement quelques années.

\section{Les lycées bilingues allemands, un modèle exportable}

40 Le traité de l'Élysée, en 1963, a été le point de départ, dans certains Länder de la République fédérale allemande, de la mise en place de sections bilingues germanofrançaises. On compte actuellement plus de cinquante établissements possédant ce type de sections bilingues, auxquels s'ajoutent des sections germano-britanniques et germanoespagnoles.

41 L'apprentissage de la seconde langue commence dès la cinquième année de la scolarité (l'équivalent du CM2) de manière assez intensive (sept séquences par semaine), mais la caractéristique essentielle du modèle est que deux ans plus tard, deux ou trois disciplines du programme allemand sont enseignées, partiellement ou totalement, en langue 2 (langue française, ou anglaise, etc.). 
Notons que l'Abitur (diplôme allemand de fin d'études secondaires) délivré à l'issue de ces sections bilingues est, dans certains cas, équivalent au baccalauréat français, donnant droit par conséquent à l'entrée à l'université française.

Ce modèle a inspiré considérablement, comme on le verra ci-après, les sections européennes françaises.

\section{Les lycées bilingues des anciens pays de l'Est : une formule originale en plein développement}

Depuis très longtemps, notamment dans l'ex-URSS, certains établissements scolaires avaient la spécialité de produire de remarquables élèves bilingues, constituant une élite, et forçant l'admiration des occidentaux.

La formule avait été un peu abandonnée. Elle semble reprendre vigueur, notamment en Russie, Pologne, Bulgarie et Hongrie.

La caractéristique essentielle est d'avoir un recrutement très sélectif (par concours, mais sans se soucier de la langue 2 visée), puis de mettre en place une "année zéro ", où les élèves apprennent la langue 2 de manière extrêmement intensive, cessant quasiment toute autre activité, tout autre apprentissage scolaire. Puis, à l'issue de cette année particulière, les élèves pratiquent l'essentiel de leurs apprentissages en langue 2.

\section{Les écoles européennes, un trilinguisme assuré}

Le modèle des neuf écoles européennes est tout à fait intéressant au plan pédagogique. Chaque école possède plusieurs sections linguistiques, poursuivant les mêmes programmes et horaires. L'enseignement est systématiquement dispensé en deux, puis trois langues. Le baccalauréat européen qui est délivré donne accès à toutes les universités de l'Union européenne. Ces écoles - en Belgique, Allemagne, Grande-Bretagne, Italie, Hollande, Luxembourg-, réservées au départ aux fonctionnaires européens mais acceptant aussi des ressortissants des pays dans lesquels elles sont implantées, ont un public international, mais aussi une équipe d'enseignants provenant de tous les pays d'Europe concernés. Ce système pluriculturel favorise considérablement l'apprentissage des langues, mais le coût élevé en empêche malheureusement une généralisation rapide.

Au-delà de ces exemples européens (il faudrait encore évoquer le cas des pays d'Europe du Nord où la langue 2 - l'anglais - est également langue d'enseignement à un moment du cursus scolaire), il nous faut évoquer également des cas de bilinguisme scolaire mis en place à grande échelle, tant en Afrique et Asie qu'en Amérique, notamment au Canada et aux États-Unis.

9 Les pays d'Afrique et d'Asie récemment décolonisés ont des systèmes éducatifs le plus souvent bilingues, la langue nationale (langue 1) se développant à côté de la langue $2 \mathrm{du}$ pays colonisateur. Malheureusement, on assiste souvent dans ces cas de figure à des difficultés, voire de graves conflits, bien compréhensibles dans les périodes de décolonisation. Les pays du Maghreb par exemple, tout en développant une légitime arabisation (langue 1) ont souvent du mal à situer le français (langue 2) dans le cursus scolaire. Il reste que le bilinguisme est un objectif actuel de ces systèmes éducatifs, ne 
serait-ce que parce que les études supérieures sont le plus souvent dispensées en langue 2.

Problèmes de même nature dans d'autres régions récemment décolonisées. C'est le cas des pays du Moyen-Orient; et c'est le cas de l'Inde ou des pays de l'Asie du Sud-Est (le Vietnam développe des écoles bilingues). Ces pays en voie de développement possèdent le plus souvent des systèmes éducatifs bilingues pour des raisons essentiellement économiques. Mais des considérations idéologiques et culturelles peuvent être en contradiction avec les logiques économiques. D'où les oscillations concernant l'introduction d'une seconde langue (le français, l'anglais) s'agissant du moment et/ou de son importance qualitative et quantitative. Les systèmes éducatifs du Maghreb illustrent parfaitement ces hésitations et fluctuations, le statut de langue 2 (ici le français) n'en finissant pas de se stabiliser.

51 Les exemples de systèmes éducatifs bilingues d'Amérique du Nord, spécifiquement au Canada (surtout) et aux États-Unis, sont aussi fluctuants et labiles pour des raisons différentes, liées à des problèmes de minorités linguistiques, et ils ont été abondamment analysés, évalués, commentés. On recense plus de mille publications concernant les expérimentations de bilinguisme scolaire en Amérique du Nord, les plus célèbres étant celles du Canada, conduites en relation avec le travail de chercheurs tels que Lambert, Cummins, Gennesee, etc.

52 La stratégie pédagogique la plus connue est alors celle de l'immersion, consistant à immerger des élèves totalement, ou partiellement, dans un système d'enseignement conduit en langue 2, langue cible, dans n'importe quelle discipline.

53 Les variantes de l'immersion sont considérables, les évaluations qui en ont été faites non moins considérables, d'où il ressort tout de même que les résultats sont globalement positifs (les enfants sont plus performants en langue 2 et langue 1 que dans le système traditionnel d'apprentissage des langues) même si des nuances et réserves doivent être faites : les résultats sont meilleurs - bilinguisme positif - lorsque la langue 2 n'est pas langue dominante de l'environnement; dans le cas inverse (lorsque la langue 2 domine la langue 1) un bilinguisme négatif peut s'installer ${ }^{10}$.

54 Ce tour d'horizon rapide concernant les situations de bilinguisme scolaire souligne l'universalité de ces situations, mais aussi leur extrême variabilité. Il illustre le fait que chaque pays, en fonction de son histoire, de son état linguistique, du statut des langues qu'il possède ou qu'il vise, de ses ressources, de la formation de ses maîtres, etc. doit trouver son propre projet linguistique. Aujourd'hui, tous les pays ou presque s'efforcent de «produire» des individus bi- ou plurilingues, pour les raisons économicosociologiques que nous avons évoquées. Mais ces raisons ne suffisent pas, et la Belgique ou la Suisse, pays officiellement plurilingues, n'ont pas de systèmes éducatifs explicitement conçus pour assurer aux élèves un bilinguisme fonctionnel.

Les politiques linguistiques des États répondent donc à des considérations qui ne sont pas toujours en faveur du bi- ou plurilinguisme, nous l'avons évoqué à plusieurs reprises. C'est sans doute une des raisons qui ont favorisé l'émergence d'un immense réseau d'établissements plurilingues de par le monde, où un enseignement dit international a pu se développer au travers de plusieurs langues. Ce réseau d'écoles (plusieurs centaines, dont le lycée international de Saint-Germain, en France, est issu) repose sur des idéaux de la Société des Nations, puis de l'Unesco, et débouche sur le baccalauréat international (BI), un baccalauréat essentiellement reconnu dans les pays anglo-saxons. On notera ici 
que ce réseau a prouvé son efficacité en matière du bi- et plurilinguisme en développant notamment (à l'instar du réseau des écoles européennes) des environnements scolaires résolument pluriculturels ${ }^{11}$.

\section{Et en France où en est-on ?} internationales des collèges et lycées : environ sept mille élèves sont inscrits en France dans ces sections. Historiquement, les sections internationales sont nées du besoin de scolariser les enfants de fonctionnaires internationaux présents sur le territoire français (comme à Saint-Germain-en-Laye par exemple avec les Supreme Headquarters Allied Powers Europe (SHAPE) de l'OTAN). L'idée qui préside à leur conception consiste à imprégner les enfants de culture française, tout en leur permettant de réintégrer sans difficulté, à tout moment, le système éducatif de leur pays. Mais actuellement, et de plus en plus, les sections internationales accueillent des enfants français (jusqu'à $75 \%$ de l'effectif). L'enseignement y est bilingue, le plus souvent au travers des programmes de littérature, d'histoire et de géographie. Les études débouchent sur le baccalauréat à option internationale (OIB) où quelques épreuves sont passées en langue 2 (avec des correcteurs étrangers). On compte plus d'une vingtaine d'établissements français ayant des sections internationales, soit que ces sections constituent l'ensemble de l'établissement - c'est le cas de Saint-Germain-en-Laye -, soit qu'elles n'en représentent qu'une partie plus ou moins réduite - c'est le cas du lycée Honoré de Balzac, à Paris. Au-delà de ces deux cas, on trouve aussi des sections internationales à Ferney-Voltaire (près de Genève), Strasbourg, Marseille, Lyon, Bordeaux, Grenoble, Valbonne (près de Nice), puis Fontainebleau et Sèvres. Les sections sont essentiellement britanniques, américaines, allemandes, espagnoles, italiennes, mais on trouve aussi, à Saint-Germain-en-Laye, des sections néerlandaise, portugaise, danoise, norvégienne, suédoise, japonaise.

Un deuxième réseau d'établissements français où l'on dispense un enseignement bilingue est constitué par les sections bilingues d'écoles, collèges ou lycées ayant intégré les langues régionales dans le cursus scolaire. De longs développements seraient nécessaires pour décrire les résultats remarquables obtenus notamment dans de très nombreux établissements publics basques, catalans, bretons, occitans ou corses, avec des stratégies diverses mais qui, dans tous les cas, au-delà de l'apprentissage et de la réhabilitation des langues régionales, aident l'élève à mieux comprendre le fonctionnement de la langue française (meilleures performances) et à apprendre plus facilement d'autres langues (mise en place d'une véritable éducation linguistique). 
61 l'écrit sur la langue allemande, langue du pays voisin et partenaire. Les sections bilingues en Alsace sont en plein développement, selon plusieurs formules, et nul doute qu'à long terme l'on ne s'achemine, dans ce cas de figure, vers un bilinguisme scolaire françaisallemand à l'échelle de la région entière. Les stratégies pédagogiques de ces sections bilingues sont diversifiées, mais partout la langue régionale (ou langue allemande pour l'Alsace) est véhiculaire, et le brevet des collèges comme le baccalauréat admettent des épreuves dans ces langues.

Un troisième réseau français est en construction depuis quelques années. Il s'agit des sections européennes dont le texte fondateur date du 19 août 1992; s'y adjoignent quelques sections dites orientales ouvertes dans les collèges et lycées à partir de la classe de quatrième. Ces sections sont actuellement au nombre de six-cents environ, et sont bilingues dans la mesure où un certain nombre de matières non linguistiques sont enseignées en seconde langue. Le modèle est celui des sections bilingues allemandes, qui datent d'une trentaine d'années. La langue 2 est le plus souvent l'anglais, parfois l'allemand, rarement l'espagnol ou l'italien. Les difficultés de mise en place sont nombreuses (manque de professeurs qualifiés notamment), mais le processus est engagé et il faut espérer que des esprits chagrins ne stopperont pas l'expérimentation sous prétexte que tout n'est pas actuellement parfait, comme ce fut le cas en 1973, lorsque le rapport Girard mit fin à toute expérimentation d'enseignement des langues à l'école élémentaire en déclarant que c'était insatisfaisant. On arrêta! Quinze ans de retard ont été pris, au lieu de tenter de remédier aux dysfonctionnements qui étaient réels.

Enfin, il existe en France un certain nombre d'écoles privées, conventionnées ou non, dont le projet pédagogique est de développer un bilinguisme scolaire. Citons, par exemple l'École active bilingue Jeannine Manuel, à Paris, mais aussi quelques écoles étrangères installées en France, comme l'American School de Paris.

Au total, un ensemble assez modeste, mais dont on peut penser qu'il va se développer, notamment grâce aux sections européennes. D'autant que les lois de décentralisation favorisent les initiatives régionales, comme on peut s'en rendre compte dans certaines académies telles que Reims ou Lille (à travers le développement d'Eurorégions); d'autant que la demande sociale est forte, et qu'il n'est plus possible de prétendre que l'enseignement bilingue rend idiot, dyslexique ou bien qu'il est responsable en lui-même d'échecs scolaires.

\section{L'enseignement bilingue, des bénéfices à trois niveaux}

En effet, à la lumière de nombreuses observations, expérimentations et analyses, et de très nombreux travaux conduits en la matière, notamment au Canada, on sait maintenant que l'enseignement bilingue est potentiellement bénéfique pour l'élève, et que si des échecs scolaires sont observés dans certains cas, ils ne sont pas dus au concept même du bilinguisme, mais bien aux conditions dans lesquelles il a été mis en œuvre. Nous envisagerons trois types de bénéfices.

Des bénéfices linguistiques d'abord: incontestablement, la seconde langue est mieux maîtrisée lorsqu'elle est utilisée pour apprendre d'autres disciplines scolaires que lorsqu'elle n'est apprise que pour elle-même. Il faut faire fonctionner ce nouvel outil de communication, cette langue 2 , il faut s'en servir, c'est assurément la meilleure manière 
d'affiner son apprentissage. Mais il y a plus : on sait maintenant que l'apprentissage et l'usage scolaire d'une seconde langue en même temps que la première, est également utile au développement d'une meilleure maitrise de celle-ci. Par exemple, les fameuses épreuves CE2 $/ 6^{\mathrm{e}}$ de français sont mieux réussies - toutes conditions égales par ailleurs au niveau des établissements, des enseignants, etc. - par des enfants en classes bilingues basques-françaises $\left(50 \% / 50 \%\right.$ ) qu'en classes monolingues françaises ${ }^{13}$.

La conscience métalinguistique que l'enseignement bilingue permet de développer explique largement ce phénomène. L'élève prend conscience (intuitivement d'abord, explicitement ensuite, pour peu que les maîtres s'engagent, s'ils le peuvent - ce qui est hautement souhaitable -, dans une perspective comparatiste) du fonctionnement des langues, donc de sa langue maternelle, et cette éducation linguistique est, parallèlement, très précieuse pour aborder une troisième langue (l'anglais ?), une quatrième, etc. C'est le «bilinguisme positif» (Lambert). Les performances en lecture ${ }^{14}$, orthographe, connaissance lexicale et syntaxique, les capacités de traduction bien sûr, sont aussi à mettre à l'actif d'un enseignement bilingue, même chez des enfants à quotient intellectuel faible ${ }^{15}$.

Des bénéfices culturels ensuite. On sait que la langue est porteuse d'une autre manière de découper le réel, qu'elle transmet des valeurs culturelles différentes, et l'enseignement bilingue ne peut qu'ouvrir aux autres, à d'autres manières de penser le monde. Il n'est pas besoin d'insister sur ce point, tant il a été développé, décrit et valorisé; et même s'il conviendrait sans doute, quelquefois, de nuancer des propos par trop optimistes, il est indéniable que l'enseignement bilingue élargit l'horizon culturel de l'élève.

Des bénéfices cognitifs enfin. On a longtemps cru (ou fait croire) que le bilinguisme freinait le développement des capacités intellectuelles des enfants, qu'il était responsable d'échecs ou de retards scolaires, qu'il générait des troubles divers (dyslexie, bégaiements), bref qu'il pouvait induire des confusions mentales (propos encore entendus dans certains milieux scolaires, ou médico-scolaires, à propos d'enfants de migrants migrants de catégorie sociale défavorisée de préférence).

Il a fallu attendre les années soixante et les nombreux travaux d'auteurs canadiens (Lambert, Peal, Genessee) pour réhabiliter l'enseignement bilingue. A partir des expériences "d'immersion", ils ont pu prouver de manière rigoureuse que, dans de bonnes conditions qu'ils ont identifiées, l'enseignement bilingue, loin de provoquer les troubles décrits ci-dessus, est de nature à favoriser le développement intellectuel d'un enfant; autrement dit, il est porteur de bénéfices cognitifs, longuement décrits par de nombreux auteurs et que l'on peut rapidement résumer de la manière suivante: le bilinguisme et l'enseignement bilingue développent des capacités d'abstraction ${ }^{16}$ (prise de conscience de l'arbitraire signifiant/signifié), des capacités à forger des concepts (exposition à des interactions linguistiques et culturelles), des compétences de traitement de l'information plus affirmées (perceptions plus vastes et plus fines, capacités d'écoute, d'attention, de vigilance), des qualités d'adaptabilité (alerte intellectuelle) mais aussi de créativité et de pensée plus ouverte et divergente.

71 Mais ces bénéfices potentiels, attendus, de l'enseignement bilingue, ne sont pas automatiques. Un certain nombre de conditions doivent être réunies pour les atteindre, que nous évoquerons maintenant, en guise de conclusion de ce rapide survol de la problématique, de l'extension et des enjeux de l'enseignement bilingue. 


\section{Le bilinguisme scolaire, l'affaire de tous}

72 La réussite de l'enseignement bilingue doit probablement autant en effet à des considérations d'environnement qu'à des stratégies pédagogiques, ces deux groupes de conditions étant bien entendu en nécessaire interaction.

\section{Les conditions d'environnement, premières et fondamentales}

73 Un bilinguisme scolaire ne peut réussir que si, sociologiquement, idéologiquement, au niveau des mentalités collectives d'un pays, se dégage l'idée que le monolinguisme n'est pas la manifestation première et obligée de l'unité et de la force d'une nation (la Suisse? les États-Unis ?), même s'il n'est pas contestable qu'une langue est souvent un puissant outil de cohésion nationale. L'histoire de nombreux pays regorge de "guerres des langues", dont on sait bien que les raisons étaient loin d'être toutes de nature patriotique, servant souvent les intérêts de groupes particuliers au plan économique et du pouvoir en général. Le bilinguisme - le bilinguisme à l'école par conséquent - ne sont pas des atteintes à la nation, et les idéologies des langues "supérieures" (de sinistre mémoire, pendant le nazisme) ne devraient plus avoir le droit de cité aujourd'hui.

Ces nécessaires évolutions des mentalités devraient pouvoir être relayées (devancées?) par les pouvoirs politiques et institutionnels. En d'autres termes, un bilinguisme scolaire ne peut vraiment fonctionner que si les autorités administratives, au plus haut niveau, prennent vraiment parti en sa faveur, et tentent de convaincre, en montrant notamment que le bilinguisme scolaire n'est pas préjudiciable au français, à la francophonie en général, et, qu'à l'inverse, le repli frileux sur une langue qui n'est plus hégémonique au plan mondial ne peut que l'affaiblir.

D'autre part, et dans le même ordre d'idées, un bilinguisme scolaire ne peut se développer que si sont vaincues un certain nombre de résistances de type corporatiste, que si les enseignants admettent que des collègues étrangers puissent travailler avec eux dans un établissement scolaire, dans le cadre d'échanges internationaux de longue durée.

En résumé, et si l'on souhaite un bilinguisme scolaire, la société et sa représentation institutionnelle doivent se doter d'une véritable politique linguistique, officielle, acceptée par le plus grand nombre de manière démocratique, et clairement volontariste, comme le suggère Claude Hagège dans un article récent ${ }^{17}$.

\section{Les stratégies pédagogiques}

Les volontés politiques et institutionnelles sont indispensables pour créer la légitimité, la dynamique, la motivation. Mais elles ne sauraient suffire, et un bilinguisme scolaire ne peut fonctionner que si des méthodologies pertinentes sont mises en place. Les formules sont diverses, comme on a pu s'en apercevoir en esquissant une typologie à l'échelle internationale. Mais dire cela ne signifie pas que toutes les formules se valent, au plan de l'efficacité. Chaque pays, en fonction de ses objectifs du moment clairement définis, de son " état linguistique ", de ses choix de langue 2 et des statuts respectifs qu'il donne aux langues 1 et 2, de son histoire, de ses ressources, de ses contraintes, de la formation de ses maitres, de son budget, doit trouver sa propre formule, son propre projet linguistique. Dans la même perspective, chaque région d'un même pays peut développer des stratégies 
différenciées en fonction de contextes différents (c'est le cas de la France, où les situations en Alsace, en Bretagne ou en Touraine ne sont pas identiques).

Des facteurs communs se retrouvent cependant dans toutes les méthodologies utilisées, et qui sont, pour l'essentiel :

- donner à la langue 2 un temps significatif, évitant par conséquent le «saupoudrage », aussi inefficace que démobilisateur;

- donner à la langue 2, en tant que langue véhiculaire, des contenus importants à apprendre, au plan scolaire, lui conférant par conséquent une dignité et un statut conséquents ;

- se doter de maitres compétents, la meilleure formule étant incontestablement un maître/ une langue, dès l'école élémentaire.

79 Mais il va de soi que ces éléments fondamentaux de réussite doivent être promus, entourés de très nombreuses mesures d'accompagnement: la formation des maîtres, notamment, est capitale, et force est de reconnaître que la France a, présentement, peu investi dans la formation linguistique de ses enseignants.

Parallèlement, il va de soi que la recherche pédagogique doit être développée, en même temps que la conception de matériels didactiques.

Des articulations doivent pouvoir exister entre les niveaux, les réseaux de bilinguisme scolaire. En France, ces échanges et coordinations n'existent guère. Quelles relations entre l'enseignement d'initiation aux langues étrangères (EILE, succédant à l'EPLV, enseignement précoce des langues vivantes) et les réseaux de sections européennes, internationales? Quelles relations entre les enseignants de langues et culture d'origine (ELCO) et ces mêmes réseaux? Des gisements importants de bilinguisme sont laissés en friche (en espagnol, arabe, portugais, turc.) à partir de l'immigration. N'est-ce pas dommage?

Au total, au plan pédagogique, l'essentiel - et le difficile - est de donner à l'élève du sens à l'apprentissage d'une seconde langue. Cette langue 2, doit avoir pour lui un statut positif, un statut valorisé et valorisant, condition indispensable pour le développement d'un bilinguisme positif.

83 Et, nous sommes renvoyés au niveau institutionnel, au niveau social. Le bilinguisme scolaire est bien en effet l'affaire de tous. La volonté d'une société.

\section{NOTES}

1. William Mackey, Bilinguisme et contact des langues, Paris, Klincksieck, 1976.

2. Josiane Hamers et Michel Blanc, Bilingualité et bilinguisme, Bruxelles, Mardage, 1983.

3. Louis-Jean Calvet, La guerre des langues et les politiques linguistiques, Paris, Payot, 1987.

4. «Les langues régionales et l'Europe ", Revue internationale d'éducation-Sèvres, n 3, 1994.

5. Pierre Calvé, « L'immersion au Canada », Études de linguistique appliquée, n 82, 1994.

6. Pierre Calvé, op. cit.

7. Aperçu sur le système d'enseignement luxembourgeois, Eurydice, 1993. 
8. Rita Decîme, « Le bilinguisme à l'école maternelle à partir de l'expérience valdotaine », Enfance, $\mathrm{n}^{\circ}$ 4, 1991, p. 311-327.

9. Enquête sur les compétences linguistiques en classe de cinquième, Aoste, IRSSAE, 1993.

10. Pierre Calvé, op. cit.

11. Jean-Pierre Maillard, "L'enseignement international en France à l'heure européenne ", Savoir-Education-Formation, $\mathrm{n}^{\circ}$ 2, avril 1991, p. 359-381.

12. Guide de l'enseignement bilingue à parité horaire, Office régional de bilinguisme, académie de Strasbourg, 1994.

13. C. Leralu, «L'enseignement bilingue dans les écoles publiques du Pays Basque français», Nouveaux Cahiers d'allemand, 1993.

14. Jean Duverger, « On n'apprend à lire qu'une fois ", Revue internationale d'éducation de Sèvres, $\mathrm{n}$ - 2, 1994, p. 73-81.

15. Fred Genesee, «L'immersion et l'apprenant défavorisé », Études de linguistique appliquée, $\mathrm{n}^{\circ} 82$, 1991, p. 77-93.

16. Daniel Coste, «Conceptualisation et alternance des langues à propos de l'expérience du Val d'Aoste ", Études de linguistique appliquée, n 96, octobre/décembre 1994, p. 105-119

17. Le Monde, 11 février 1995.

\section{RÉSUMÉS}

À une définition univoque $d u$ concept d'enseignement bilingue correspondent, à travers le monde, des réalités très diverses. L'auteur en dresse un panorama et propose, pour en apprécier les enjeux, d'inventorier les variables, leurs combinatoires, les liens entre contexte local et dispositif choisi : sous la multiplicité des formes, les conditions à réunir paraissent homologues.

\section{INDEX}

Index géographique : France, Pays basque, Alsace, Allemagne, Italie, Val d'Aoste, Espagne, Catalogne, Pays-Bas, Luxembourg, Russie, Pologne, Bulgarie, Hongrie, Canada, Québec, ÉtatsUnis, Suisse, Tunisie, Grande-Bretagne, Belgique, Vietnam

Mots-clés : analyse comparative, enseignement bilingue, section bilingue, enseignement des langues, apprentissage d'une langue étrangère

\section{AUTEUR}

\section{JEAN DUVERGER}

Direction générale des relations culturelles, scientifiques et techniques, ministère des Affaires étrangères, Paris, France 